\title{
A fast and simple LC-MS-based characterization of the flavonoid biosynthesis pathway for few seed(ling)s
}

Benjamin Jaegle ${ }^{1 \dagger}$, Miran Kalle Uroic ${ }^{1 \dagger}$, Xu Holtkotte $^{1}$, Christina Lucas ${ }^{1}$, Andreas Ole Termath ${ }^{2}$, Hans-Günther Schmalz ${ }^{2}$, Marcel Bucher ${ }^{1}$, Ute Hoecker ${ }^{1}$, Martin Hülskamp ${ }^{1 *}$ and Andrea Schrader ${ }^{1 *}$

\begin{abstract}
Background: (Pro)anthocyanidins are synthesized by the flavonoid biosynthesis pathway with multi-layered regulatory control. Methods for the analysis of the flavonoid composition in plants are well established for different purposes. However, they typically compromise either on speed or on depth of analysis.

Results: In this work we combined and optimized different protocols to enable the analysis of the flavonoid biosynthesis pathway with as little as possible biological material. We chose core substances of this metabolic pathway that serve as a fingerprint to recognize alterations in the main branches of the pathway. We used a simplified sample preparation, two deuterated internal standards, a short and efficient LC separation, highly sensitive detection with tandem MS in multiple reaction monitoring (MRM) mode and hydrolytic release of the core substances to reduce complexity. The method was optimized for Arabidopsis thaliana seeds and seedlings. We demonstrate that one Col-0 seed/seedling is sufficient to obtain a fingerprint of the core substances of the flavonoid biosynthesis pathway. For comparative analysis of different genotypes, we suggest the use of 10 seed(lings). The analysis of Arabidopsis thaliana mutants affecting steps in the pathway revealed foreseen and unexpected alterations of the pathway. For example, HY5 was found to differentially regulate kaempferol in seeds vs. seedlings. Furthermore, our results suggest that COP1 is a master regulator of flavonoid biosynthesis in seedlings but not of flavonoid deposition in seeds.
\end{abstract}

Conclusions: When sample numbers are high and the plant material is limited, this method effectively facilitates metabolic fingerprinting with one seed(ling), revealing shifts and differences in the pathway. Moreover the combination of extracted non-hydrolysed, extracted hydrolysed and non-extracted hydrolysed samples proved useful to deduce the class of derivative from which the individual flavonoids have been released.

Keywords: Anthocyanidin, Proanthocyanidin, Flavonoids, Seed, Seedling, Deuterated internal standard, LC-MS, Hydrolysis, Arabidopsis thaliana

Abbreviations: (e-)f3-ol, (epi-)flavan-3-ol; ANR, Anthocyanidin reductase; ANS, Anthocyanidin synthase; BF3, Boron trifluoride; bHLH, Basic-helix-loop-helix; BuOH, Butanol; c, Catechin; CHI, Chalcone synthase; CHS, Chalcone syntase; COP1, Constitutive photomorphogenic 1; cps, Counts per second; cy, Cyanidin; d, Deoxy-hexose; D3-q, D3-quercetin; D3-s, D3-sakuranetin; de, Delphinidin; DFR, Dihydroflavonol 4-reductase; e, Epicatechin; EIC, Extracted ion chromatogram; (Continued on next page)

\footnotetext{
* Correspondence:

martin.huelskamp@uni-koeln.de; andrea.schrader@uni-koeln.de

${ }^{\dagger}$ Equal contributors

'Botanical Institute and Cluster of Excellence on Plant Sciences (CEPLAS),

University of Cologne, Cologne Biocenter, Zülpicher Str. 47b, 50674 Cologne,

Germany

Full list of author information is available at the end of the article
} 


\begin{abstract}
(Continued from previous page)
ESI, Electrospray ionization; F3'5'H, Flavonoid 3',5' hydroxylase; F3'H, Flavonoid 3'-monooxygenase; F3H, Flavanone 3hydroxylase; FA, Formic acid; FLS, Flavonol synthase; GST, Glutathione S-transferase; h, Hexose; HCl, Hydrochloric acid; HPC, High precision calibration; HY5, Elongated hypocotyl 5; is, Isorhamnetin; ka, Kaempferol; ka-DIL, Kaempferol-diluted; LAR, Leucoanthocyanidin reductase; LC, Liquid chromatography; LDOX, Leucoanthocyanidin dioxygenase; LOD, Limit of detection; LOQ, Limit of quantification; MBW, MYB/bHLH/WD40; MeOH, Methanol; MRM, Multiple reaction monitoring; MS, Mass spectrometry; my, Myricetin; MYB, V-myb myeloblastosis viral oncogene homolog; na, Naringenin; NMR, Nuclear magnetic resonance spectroscopy; OMT, O-methyltransferase; PAP, Production of anthocyanin pigment; pB, Procyanidin B2; pe, Pelargonidin; qu, Quercetin; RNAi, RNA interference; RSD, Relative standard deviation; S/N, Signal-to-noise ratio; SPA1, Suppressor of phytochrome A-105; ta, Taxifolin; TFA, Trifluoroacetic acid; THF, Tetrahydrofuran; TOF, Time-of-flight; TT, Transparent testa; TTG1, Transparent testa glabra 1; WD40 domain, Consists of WD40 repeats; WD40 repeat, Usually consists of about 40 amino acids often ending with W-D (tryptophan-aspartic acid).
\end{abstract}

\section{Background}

Flavonoids play a role in a wide variety of biological phenomena. In plants, their bright colors attract pollinators while their antioxidant properties offer protection from harmful UV-radiation. For humans, proanthocyanidins from red wine have been discussed to explain the "French paradox" - the co-occurrence of low coronary heart disease deaths and a diet rich in saturated fat [1-3]. In particular the medical implications led to extensive studies on their uptake, metabolism and excretion in animals and humans $[4,5]$. Flavonoids are phenylpropanoid-derived secondary metabolites that may accumulate in various plant tissues. Their production is often regulated by environmental factors including light, temperature, pathogen attack and nutrient deprivation. Flavonoids represent a complex group of compounds. The major subgroups comprise chalcones, flavones, flavonols, flavandiols, anthocyanidins and proanthocyanidins [6].

As most structural genes of the pathway are monogenic in Arabidopsis thaliana (A. thaliana) [3], this model species is well suited to analyse the flavonoid core biosynthesis. The underlying genetic loci of structural and regulatory genes were mainly derived from mutant screenings for reduced seed coat pigmentation and were initially named TRANSPARENT TESTA (TT) [7, 8].

CHALCONE SYNTHASE (CHS/TT4) catalyses the first committed step of the pathway (Fig. 1a): the synthesis of chalcone. Chalcone is isomerized to naringenin by CHALCONE ISOMERASE (CHI/TT5). Naringenin and eriodyctiol are branching points to other flavonoid classes. FLAVANONE 3-HYDROXYLASE (F3H/TT6) converts naringenin to the first compound of the next level in the plant's flavonoid biosynthesis. This level comprises the 3-OH flavanones dihydrokaempferol, taxifolin and dihydromyricetin which are interconverted by FLAVONOID 3'MONOOXYGENASE (F3'H, TT7) and FLAVONOID 3,5' HYDROXYLASE (F3'5'H). All three substances serve as educts for FLAVONOL SYNTHASE (FLS) and DIHYDROFLAVONOL 4-REDUCTASE (DFR/TT3) resulting in three further branches of the pathway. The flavonols kaempferol, quercetin and myricetin, are the products of FLS. The leucoanthocyanidins leucopelargonidin, leucocyanidin and leucodelphinidin are synthetized by DFR and further converted to the anthocyanidins pelargonidin, cyanidin and delphinidin by LEUCOANTHOCYANIDIN DIOXYGENASE (LDOX/TT11/17/18) and to the epi-flavan-3-ols epiafzelechin, epicatechin and epigallocatechin by ANTHOCYANIDIN REDUCTASE (ANR). LEUCOANTHOCYANIDIN REDUCTASE (LAR) catalyses the synthesis of the flavan-3-ols afzelechin, catechin and gallocatechin from leucoanthocyanidins. Modification by methylation is catalysed by $\mathrm{O}$ METHYLTRANSFERASEs (OMTs) e.g. quercetin to isorhamnetin, cyanidin to peonidin and delphinidin to petunidin and malvidin. Multimerization and further modification of epi- and flavan-3-ols results in proanthocyanidins and condensed tannins [9]. Flavonols and (pro)anthocyanidins are the major metabolic sink of the flavonoid biosynthesis pathway [10]. After synthesis, flavonoids - mainly flavonols and anthocyanidins - are subjected to multiple successive modifications through glycosyl-, methyl- and acyltransferases to give rise to a plethora of derivatives [11]. These modifications are necessary for a stable storage of anthocyanidins in planta [12].

In particular the analysis of $A$. thaliana mutants has revealed a multi-layered regulation for the key enzymes of the flavonoid biosynthesis pathway [1315]. Embedded in light signalling, CONSTITUTIVE PHOTOMORPHOGENIC 1 (COP1)/SUPPRESSOR OF PHYTOCHROME A-105 (SPA) complexes mediate posttranslational degradation of light-regulated transcription factors like ELONGATED HYPOCOTYL (HY5) and PRODUCTION OF ANTHOCYANIN PIGMENT 2 (PAP2) which accumulate in cop1 mutants and transcriptionally activate multiple enzymes of the pathway [16-21]. The COP1 protein is inactivated by light and is therefore mainly active in darkness where it suppresses photomorphogenesis [22, 23]. It is also 


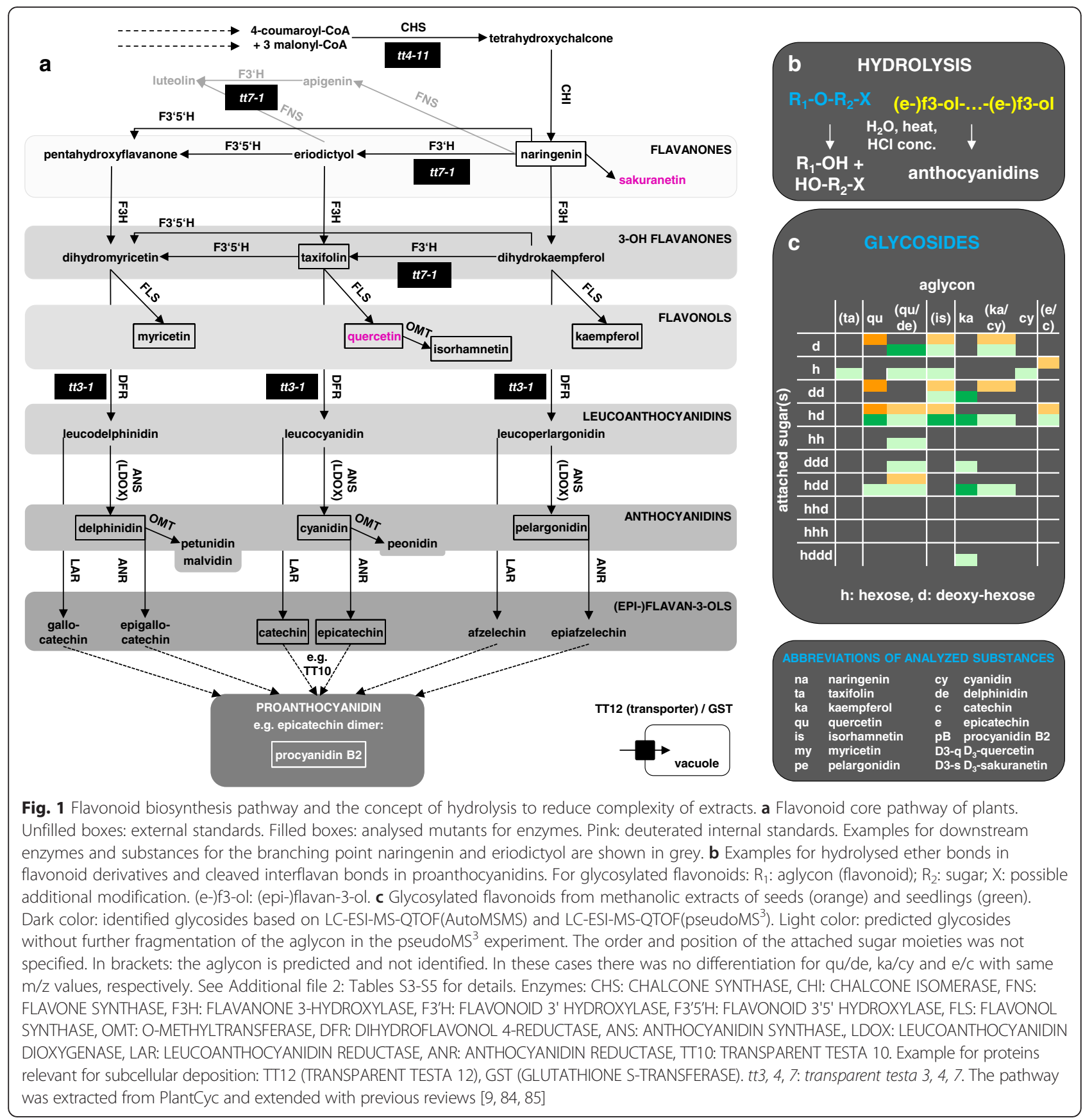

relevant for the regulation of the circadian clock and photoperiodic flowering [24].

Most knowledge of the regulation of anthocyanidin biosynthesis as part of photomorphogenesis by COP1 and HY5 has been derived from studies on protein stability, genetic analysis of mutants, studies on changes in gene expression in mutants and photometric anthocyanidin measurements.

The expression profile of dark-grown cop1 mutants is similar to light grown wild-type seedlings explaining the constitutive photomorphogenetic phenotype of these mutants. In cop1 mutant seedlings, e.g. increased levels of CHS, CHI, FLS1 and F3H were reported [21, 25]. CHS expression serves as one of the markers for COP1-dependent photomorphogenesis. Although COP1 and HY5 act in an antagonistic manner, many HY5-regulated genes overlap with the group of COP1-regulated genes [21]. HY5 activates the expression of early and late anthocyanidin biosynthesis genes (CHS, CHI, F3H, F3'H, DFR and $L D O X)$ by directly binding to the promoters of these genes in seedlings [16].

DFR expression can also be activated by PAP1 and PAP2 [26]. PAP2 can join V-myb myeloblastosis viral 
oncogene homolog (MYB)/basic-helix-loop-helix (bHLH)/ WD40 (MBW) complexes. In the TRANSPARENT TESTA GLABRA 1 (TTG1)-MBW complexes the WD40 protein TTG1 acts together with combinations of different MYB and bHLH proteins to transcriptionally regulate downstream genes [27]. The central role of TTG1 led to a classification of early and late parts of the flavonoid biosynthesis pathway [28] such that late steps are TTG1MBW-dependent [29-31].

Not only the amount of flavonoids is subjected to regulation but also tissue-specific composition. In $A$. thaliana seeds, mainly epicatechin, proanthocyanidins and quercetin-based glycosides are detected whereas in leaves kaempferol-based glycosides and anthocyanidins dominate [31-34]. Widely differing compositions were reported between species [35].

A central aspect in all studies is the chosen methodology for the analysis of the flavonoid composition. Methods compromise in many respects: the extraction method determines efficiency of substance recovery and modification. The analysis typically compromises on speed, sensitivity and depth of detail for the substances. Due to chemodiversity, the extraction efficiency of substances with differing polarities depends on the solvent [36]. For polar and semipolar substances $\mathrm{MeOH} /$ water is used and apolar substances are extracted with chloroform [37]. Using $\mathrm{MeOH} /$ water, glycosylated flavonoids are mainly extracted from seeds in the soluble fraction and condensed tannins occur in the non-extracted fractions. Typically, both are subsequently hydrolysed and subjected to photometrical measurement for quantitative comparisons [33]. Ether cleavage is catalysed under acidic conditions combined with heat [38]. The efficiency of hydrolysis is influenced by acidity, temperature and time of hydrolysis. Multiple substances like ferric agent, TFA, butanol, methanol and $\mathrm{HCl}$ have been used for hydrolysis of plant extracts [33, 39, 40]. Hydrolytic conditions do not only release aglycons but also cleave the interflavan bonds of proanthocyanidins eventually leading to the release of anthocyanidins from proanthocyanidins $[39,41]$.

Analysis of natural products has been highly facilitated by improvements of LC-MS detection techniques. Multiple reaction monitoring (MRM), a mode in tandem mass spectrometry, provides high selectivity and sensitivity to lower thresholds of detection. Time-of-flight detectors allow the identification of single metabolites due to precise ion traces [42]. The identity of nonhydrolysed glycosylated flavonoids can be determined through neutral loss analysis employing e.g. pseudoMS ${ }^{3}$ following chromatographic separation [43-46]. For a more precise determination, fragmentation patterns and isolated substances may be additionally analysed by NMR [42]. Meanwhile the combination of metabolomics and transcriptomics has been successfully used for decoding gene functions and to analyse the diversity of the pathway [11, 47-49].

Here we describe a method that aims to facilitate high-throughput studies analysing the flavonoid biosynthesis pathway in a reasonable time frame with sufficient precision and sensitivity to obtain a fingerprint of core components. Towards this end we: 1 ) provide a robust simple extraction and analysis protocol, 2) established external and deuterated internal standards enabling the unambiguous identification of selected core compounds, 3) reduced the number of biological material (i.e. seeds), and 4) revealed shifts and differences of the pathway in a set of mutants as a proof of principle.

\section{Results}

\section{Optimizing for high throughput}

For optimizing this method for high throughput analysis we considered four aspects: 1 . minimizing the time for LC-MS runs and data management, 2 . covering a maximum of selected substances at quantifiable levels, 3 . minimizing experimental error and 4. reducing the amount of plant material.

The first point is achieved by using the highly selective and sensitive MRM mode in tandem mass spectrometry, which produces data files of small size in combination with a short LC gradient. For quality control purposes two MRMs were selected per reference substance (Additional file 1: Table S1): the quantifier (underlined in Additional file 1: Table S2) and the qualifier (to assure that the correct compound is detected). For separation we used a short column with small particle size based on core-shell technology (KINETEX $2.6 \mu \mathrm{m} \mathrm{C} 18100 \AA$ (4.6 mm x $50 \mathrm{~mm}$ ) C18 column from phenomenex) leading two a high peak resolution at short runtime. The shortest time allowing separation of MRM-peaks for all reference substances was selected. In this study catechin/ epicatechin separation was limiting. This setup required only $13 \mathrm{~min}$ for LC-MS per sample which corresponds to 80 samples per day including all controls.

Guided by aspects two to four, the remaining parameters were optimized using $A$. thaliana seeds.

\section{Concept of hydrolysis}

In $A$. thaliana, many of the flavonoid molecules are found in various glycosylated forms or are deposited as condensed tannins [11]. As expected, without hydrolysis, we found a complex mixture of compounds resulting in various overlapping peaks using the LC-MS-QTOF set up. Consistent with previous studies we identified predominantly quercetin-based glycosides in seeds and mainly kaempferol-based glycosides in seedlings (Fig. 1c, Additional file 2: Tables S3-S6) [33, 34, 50]. 
To reduce the complexity of metabolites in seed extracts, we included a hydrolysis step releasing a fingerprint of extracted (e.g. glycosylated flavonoids) and non-extracted (e.g. condensed tannins) substances (Additional file 2: Figure S1). The ether linkage through which modifications are attached to many flavonoids (in most cases containing a glycoside) or the interflavan bond through which multimers are formed (proanthocyanidins) are cleaved under hydrolytic conditions and releases aglycons from glycosylated flavonoids as well as anthocyanidins from proanthocyanidins (Fig. 1b) [11, 33, 39].

\section{External and internal standards}

A set of 11 aglycons and procyanidin B2 was selected as external standards representing different levels of the core pathway (Fig. 1a). These standards cover several levels of the flavonoid biosynthesis pathway in A. thaliana from naringenin to kaempferol or taxifolin, either to quercetin and isorhamnetin or to cyanidin and further through epicatechin to a proanthocyanidin like procyanidin B2 [51]. In addition we used the flavonol myricetin, the anthocyanidins delphinidin and pelargonidin, and catechin, the epimer of epicatechin (Additional file 1: Table S1, Additional file 3: Figure S2).

In order to analyse extracted and non-extracted hydrolysates, two internal standards were required withstanding the (two-step) extraction procedure. Because we aimed to develop a method adaptable to a wide range of species, we synthesized two substances that are normally not found in plant extracts: $\mathrm{D}_{3}$-quercetin and $\mathrm{D}_{3}$-sakuranetin (Additional file 3: Methods S1). MRMs were selected (Additional file 1: Table S2) and both standards were tested for linearity (Additional file 3: Figure S3). Concentrations used in this study are in the linear range of the respective internal standard. For $\mathrm{D}_{3}$-quercetin, we observed a non-significant decrease to $76 \%(+/-23 \%)$ of its response over time in the presence of acid (1\%FA) relative to non-acidified $\mathrm{MeOH}(92 \%+/-15 \%)$ when left for $24 \mathrm{~h}$ at $5^{\circ} \mathrm{C}$ in the sample taker (Additional file 3: Table S7). Relative to the initial response, the response of $\mathrm{D}_{3}$-sakuranetin was neither changed by acid nor over time with a response of $100 \%(+/-23 \%)$ and $95 \%(+/-$ $15 \%)$ with and without $1 \%$ FA.

The external standards were analysed in a range of 1 to $1000 \mathrm{nM}$ and normalized with $\mathrm{D}_{3}$-sakuranetin (Additional file 3: Tables S7 and S8). All components were detectable in a linear range with a mean relative standard deviation (RSD) of $14.5 \%$. Only values for myricetin at a concentration of $1 \mathrm{nM}$ were excluded. Comparing the standards in $\mathrm{MeOH}$ or $\mathrm{MeOH}+1 \% \mathrm{FA}$ at 0 and $24 \mathrm{~h}$ at $5^{\circ} \mathrm{C}$ (Additional file 3: Table S7) revealed that in acidified $\mathrm{MeOH}$ most substances were stable. Therefore, $\mathrm{MeOH}+1 \% \mathrm{FA}$ was used for the quality control.

\section{Extraction and hydrolysis time}

The selected extraction protocol was modified from an extraction protocol previously used for seeds [33]. We simplified the protocol to allow robust high throughput LC-MS application by extracting with aqueous, acidified $\mathrm{MeOH}(1 \% \mathrm{FA})$ and by hydrolysis with hydrochloric acid in $\mathrm{MeOH}$ instead of $\mathrm{BuOH} / \mathrm{HCl} /$ ferric agent (e.g. [33, 39]). We added $1 \%$ of formic acid (FA) to stabilize extracted anthocyanidins as they are known to be $\mathrm{pH}$ sensitive [12]. From a wide spectrum of solvents and combinations thereof used for flavonoid extraction [33, $39,52-54]$, we decided to use a concentration of $50 \%$ $\mathrm{MeOH}$ selected from the optimal window of 30-50\% $\mathrm{MeOH}$. Except for naringenin and myricetin, this solvent allowed the detection of all other substances derived from our set of compounds at quantifiable levels when using ten $A$. thaliana seeds (Additional file 4: Figures S4, S5 and Table S9).

Extracted non-hydrolysed samples contained minor amounts of free core substances (e.g. epicatechin and procyanidin B2), while extracted, hydrolysed samples contained mainly the released aglycons. In non-extracted hydrolysates, released anthocyanidins represent the content of the non-extracted condensed tannins from which they are released [39]. We subjected all three types of extracts to LC-MS analysis and accurate-mass analysis revealed a reduced complexity of hydrolysed samples (Additional file 2: Figure S1).

In previous studies, a hydrolysis time of $60 \mathrm{~min}$ was used $[33,52]$. In the next step, we aimed to reduce this hydrolysis time to minimize the degradation of core substances while completely hydrolysing the most abundant glycosylated flavonoids. Three quercetin based glycosides dominate extracted non-hydrolysed samples in LC-ESIMS-QTOF(pseudoMS ${ }^{3}$ ) analysis (Fig. 2a). After twenty minutes of hydrolysis, none of these were detectable anymore (Fig. 2b). Longer hydrolysis led to reduced epicatechin levels (Additional file 4: Figure S6 and Table S10). Therefore, we used a hydrolysis time of 20 min for the extractable fraction. For the nonextractable fraction, a shorter application of hydrolytic conditions of $10 \mathrm{~min}$ proved to be optimal (Fig. 2c, Additional file 4: Figure S6).

\section{Effect of hydrolysis on reference substances}

In hydrolysed samples, the method described in this study aims to compare whole branches of the pathway between mutants or ecotypes to reveal major shifts. This facilitates the identification of parts of the pathway that can be analysed in more detail. Therefore, the method uses core substances of the flavonoid pathway analysed in hydrolysed samples as representatives for groups of substances, namely all substances from which they can be released by hydrolysis. 

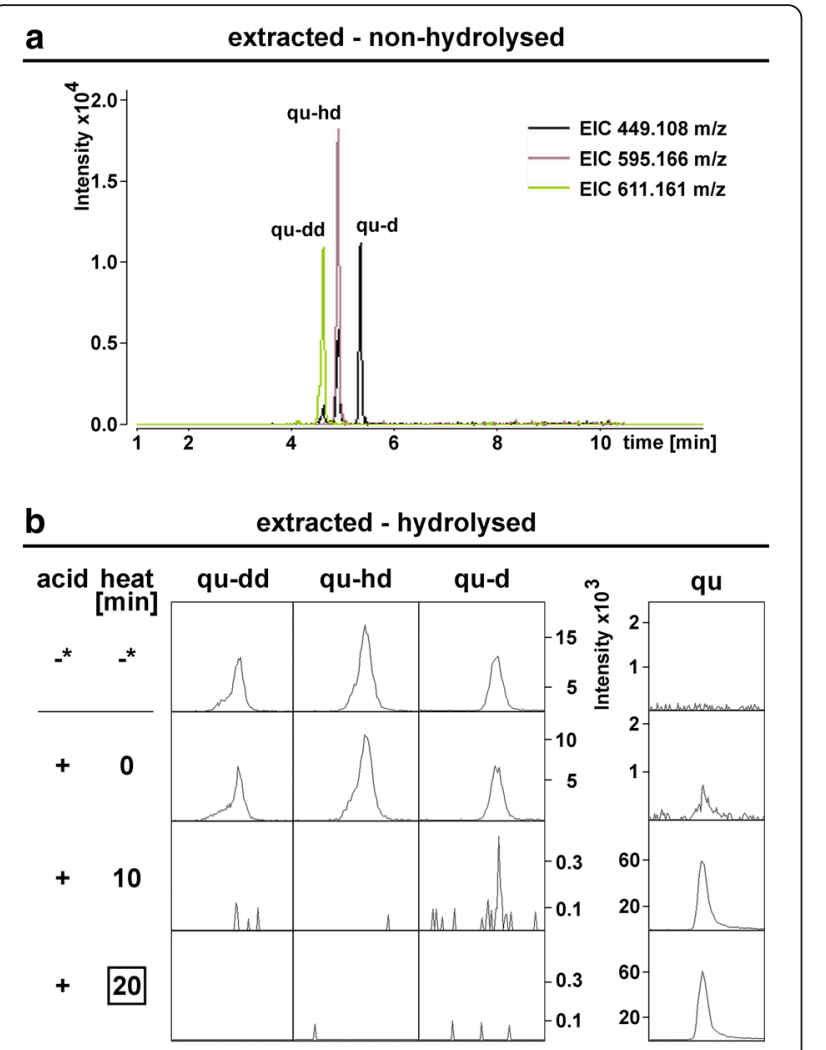

c

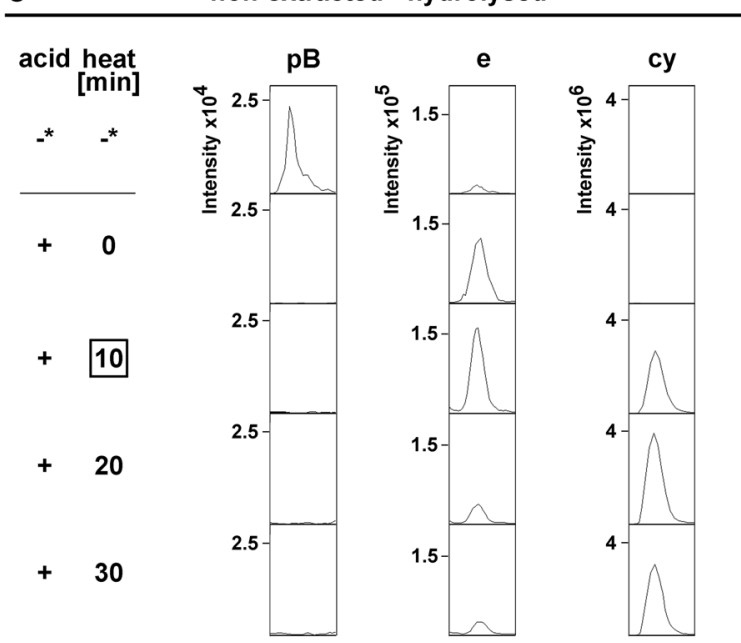

Fig. 2 Selection of hydrolysis time for seeds. LC-ESI-MS-QTOF (AutoMSMS) analysis of non-hydrolysed Col-0 seed samples $(n=10)$. a Merged EICs for quercetin-bases glycosylated flavonoids with the strongest response. $\mathbf{b}$ Extracted non-hydrolysed samples before (-) and after addition of acid $(+)$ and heat treatments. c non-extracted samples after addition of acid (+) and heat treatments. *: extracted non-hydrolysed samples are shown for comparison. acid: $\mathrm{HCl}$ in $\mathrm{MeOH}$. Boxed: selected hydrolysis time derived from this experiment. See Additional file 4: Figure S6 and Table S10
We tested for each of the selected substances the corresponding external standards separately through hydrolysis for possible degradation or conversions. $t t 4-11$ seeds were spiked with the respective substance prior to extraction (Additional file 5: Figure S7). For comparison, the respective standards were diluted with the same factor as introduced through extraction and treatment using $\mathrm{MeOH}+/$-FA (according to the respective stability, see Additional file 3: Table S7).

All substances - except procyanidin B2 - withstand both hydrolysis protocols. The procyanidin B2 standard (90\% purity) releases epicatechin and cyanidin at quantifiable levels. In hydrolysed samples, no procyanidin B2 is detectable. The release of cyanidin from procyanidin under hydrolytic conditions has been widely used before to estimate procyanidin levels [39]. Despite a conversion from procyanidin $\mathrm{B} 2$, epicatechin might also be released from impurities in the standard.

Furthermore, we found that epicatechin can be converted into catechin (Fig. 3, tt4-11+epicatechin). Catechin is not detectable in non-hydrolysed Col-0 seed samples but found in hydrolysed samples indicating that epiconversion occurs during hydrolysis. Therefore, no conclusions on the presence of catechin can be drawn from hydrolysed samples. Surprisingly, myricetin, pelargonidin and delphinidin released quantifiable amounts of catechin but not epicatechin under hydrolytic conditions for extracted samples. One possible explanation for this observation could be impurities in the standard.

Pelargonidin was detectable from all extracted hydrolysed samples including the sample only containing the internal standard (Additional file 5: Figures S7, S8a-c). No detectable release of pelargonidin was observed from non-extracted hydrolysed samples. This indicates that low amounts of pelargonidin are released from $\mathrm{D}_{3}$-quercetin under hydrolytic conditions in the extracted samples. Therefore, minor levels of pelargonidin are not considered when interpreting results from extracted and subsequently hydrolysed samples.

In addition to these results, we cannot exclude that compounds generated downstream of CHS/TT4 which are not represented in our set of standards release one of our selected core substances under hydrolytic conditions from seeds with an intact CHS/TT4 gene.

\section{Minimizing the number of seeds}

Previous studies and specific extraction protocols require up to 200 seeds or even more [33, 52]. However, seed material is often limiting and genetic analysis may require the analysis of a particular genotype in many replicates. We therefore aimed to adopt the method to a minimum number of $A$. thaliana Col-0 seeds.

With one seed, we were able to detect in at least three of five samples kaempferol, quercetin, isorhamnetin, 

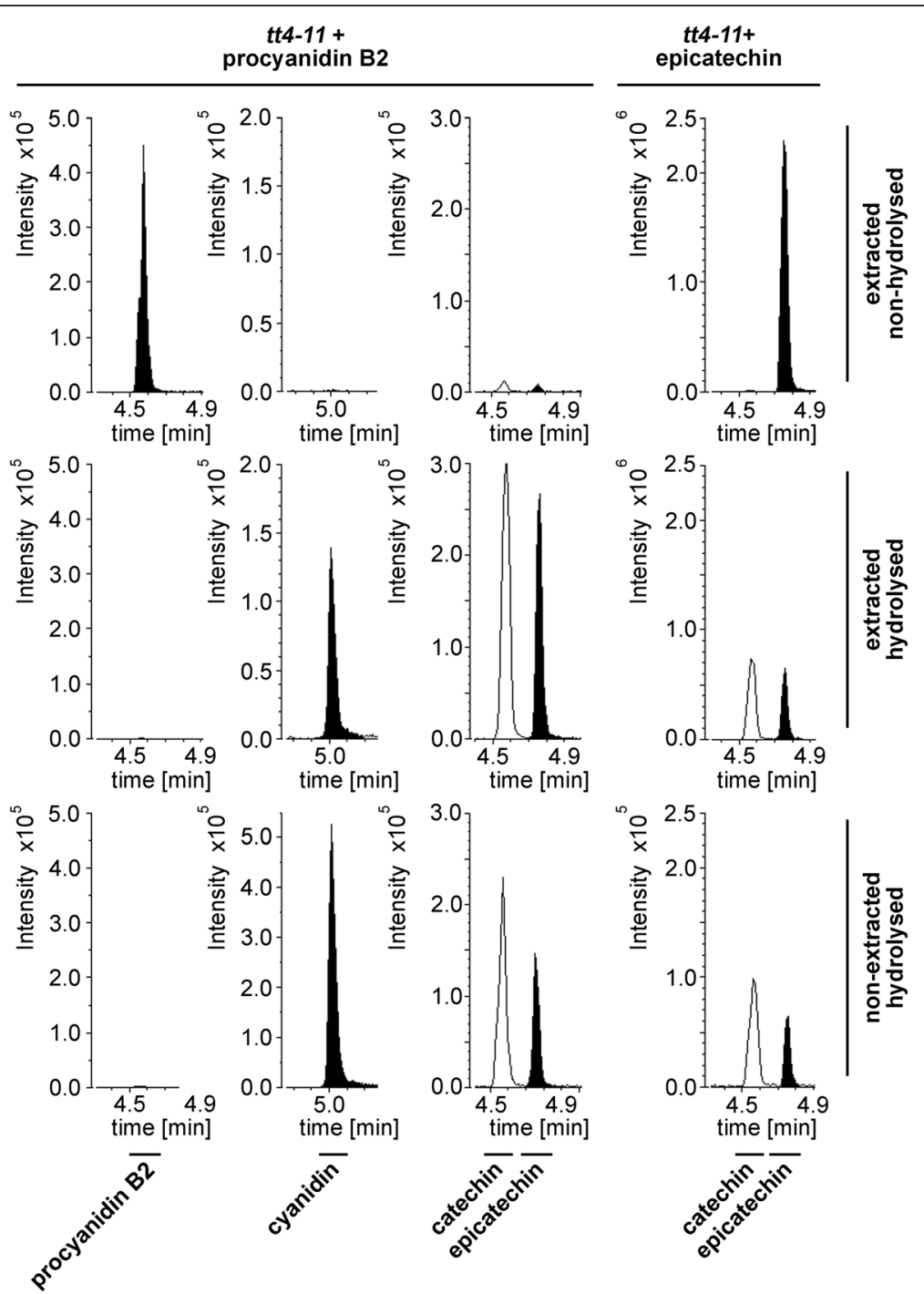

Fig. 3 Epimerization of epicatechin to catechin. Analysis of the conversion of procyanidin B2 and epicatechin after different treatments by LC-ESIMS-QTRAP(MRM). The above indicated substances were spiked on ten tt4-11 seeds prior to extraction (extracted samples) or prior to hydrolysis (non-extracted samples). Shown are MRMs for the substances indicated below the chromatograms

cyanidin and epicatechin/catechin at quantifiable levels (>LOQ) which represent the most abundant released flavonoids from seeds (Fig. 4a, Additional file 6: Table S11). In the experiment shown in Fig. 4a, few substances were close to their respective threshold of quantification for specific seed numbers. In a replicate experiment, for example, taxifolin was quantifiable in five seeds in contrast to kaempferol (Additional file 6: Figure S9). Although detectable at levels passing the LOQ in extracted hydrolysed samples from one seed, the RSD ranged between 20 to $50 \%$ for the above named six substances (Additional file 6: Table S11). Over all, most substances were detectable at quantifiable levels in the linear range when analysing the extracted fraction from five to 25 seeds (Fig. 4b, Additional file 6: Table S11). To achieve this with the non-extracted fraction, ten to 50 seeds could be used. Consequently, we used ten seeds for all subsequent experiments.

Pelargonidin was detectable but not quantifiable in the whole range from one to 25 seeds in extracted hydrolysed samples which is explained by its release from $\mathrm{D}_{3^{-}}$quercetin.

Unexpectedly, we detected delphinidin in hydrolysed samples. This is surprising as no F3'5' $\mathrm{H}$ gene is present in A. thaliana and the detection of delphinidin has not been reported for $A$. thaliana. One possible explanation is the conversion from flavonoids not covered by our reference set. Alternatively, it is possible that delphinidin is released from derivatives in extracted and nonextracted fractions. In this context, it might be relevant 

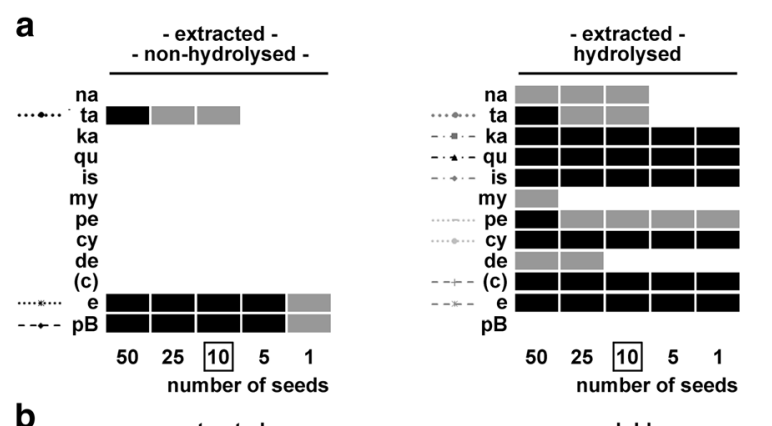

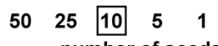
number of seeds - soluble -

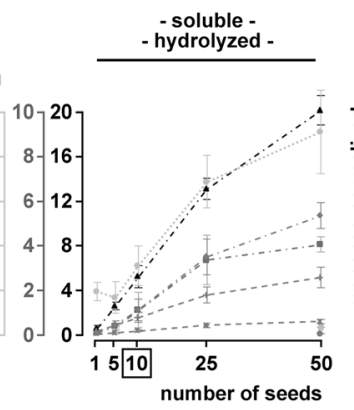

extracted

C

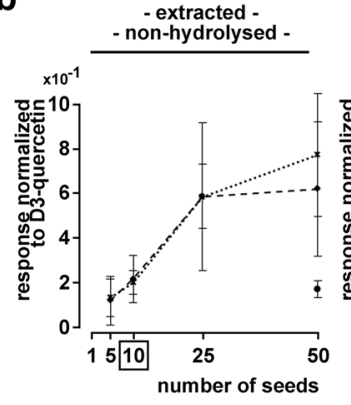

- extracted -

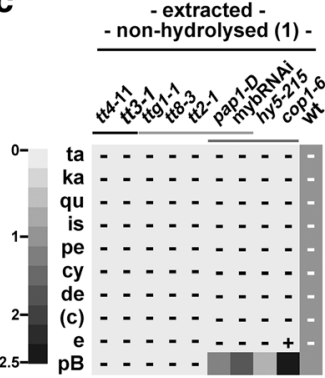

d

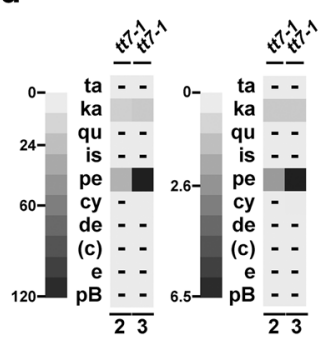

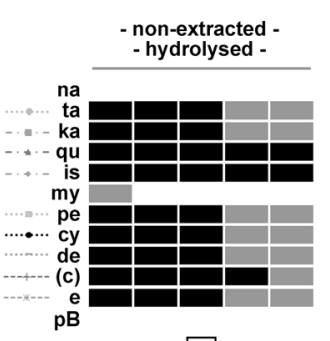

$\begin{array}{llll}50 & 25 \quad 10 & 5 & 1\end{array}$ number of seeds
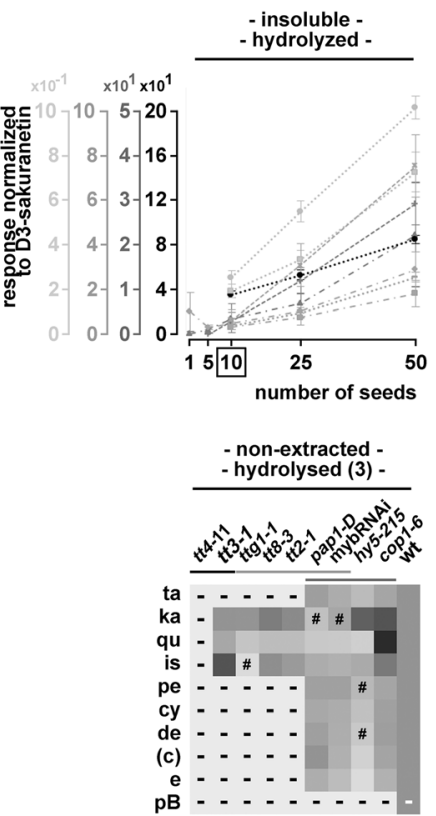

Fig. 4 Reduction of seed material and proof of principle with seeds. Reduction of seed material down to one seed (a,b) and application to a set of selected mutants (c-e) with extracted non-hydrolysed, extracted hydrolysed and non-extracted hydrolysed samples analysed by LC-ESI-MSQTRAP(MRM). a Flavonoids with detectable and quantifiable responses in 50, 25, 10, 5 and 1 A. thaliana Col-0 seed(s). Grey: LOD but not LOQ is passed, black: LOQ is passed for the majority of replicates. See also Additional file 6: Figure S9 and Additional file 5: Figure S7 and S8 (effect of hydrolysis on analysed flavonoids). b Responses normalized with the respective deuterated internal standard. Several scales are used for each subfigure. Grey values of scales correspond to the grey values of the substances. Boxed: optimal seed number according to this experiment. Error bars = STDEV. (Additional file 6: Table S11: statistics) $\mathbf{c}$-e) Mutant analysis: Heatmaps showing $\log _{2}$ of fold differences relative to the respective wild types. d Same as in (c) and (e) for tt7-1 with appropriate scales. e Same data as in (c) but normalized with kaempferol. -: LOD not passed, \#: LOD but not LOQ passed, +: LOQ passed for the mutant but not for the wild type. Lines in grey shades group the three sets of mutants: enzymes, TTG1-MBW complex components, light signalling mutants. Additional file 7: Tables S12 and S13: statistics. Additional file 7: Figure S10: responses normalized with respective deuterated internal standard. c Detected catechin which is most likely derived from epimerization of epicatechin (see Fig. 3) 
that delphinidin is fairly unstable over time, decreases with increased hydrolysis time (Additional file 3: Table S7, Additional file 4: Figure S6 and Table S10) and that our reduced hydrolysis time enables the delphinidin detection.

\section{Proof of principle: revealing shifts in the pathway}

As a proof of principle for revealed metabolic shifts and differences in the pathway, we applied the method to a set of mutants affected in different steps of the flavonoid pathway.

Two types of mutants with different effects on the pathway were analysed: First, defective or absent enzymes completely block whole branches or the entire pathway. Second, defective transcriptional regulators affect one or multiple enzymes of the pathway. We selected mutants defective in the enzymes CHS, F3' $\mathrm{H}$ and DFR (tt4-11, tt7-1 and $t t 3-1)$, mutants in transcriptional regulators of the pathway including TTG1, TT8 and TT2 (that block the same branch of the pathway as mutants of $D F R / T T 3$ ) and light signalling mutants of COP1, HY5, a PAP1 overexpressor line and a RNAi line repressing PAP1 through 4 (PAP1, PAP2, MYB113 (PAP3), MYB114 (PAP4) [28, 55].

Shifts and blockages within the pathway were observed in all three selected $A$. thaliana mutants affecting enzymes (tt4-11, tt7-1 and tt3-1) (Fig. 4c,d, Additional file 7: Figure S10), which is in agreement with previous studies $[33,56]$. In $t t 7-1$ the observed shift to the kaempferol/pelargonidin branch was reported before and is clearly visualized (Fig. 4d) [33]. To our surprise we also detected cyanidin at quantifiable levels in $t t 7-1$ extracts after hydrolysis. A close analysis of chromatograms, however, revealed that hydrolysis releases small amounts of cyanidin from the pelargonidin reference substance (Additional file 5: Figure S8d,e). Thus, quantitative analysis of hydrolysed extracts with high pelargonidin level requires the adjustment of thresholds for cyanidin quantification.

The MBW complex components TTG1, TT8 and TT2 are known to be essential regulators of $D F R[27,57]$. In agreement with this, no products of late enzymes were detected in the three respective mutants as described before (e.g. [33]). The block at DFR in tt3-1 mutants led to a strong increase of isorhamnetin and slightly elevated kaempferol levels while quercetin levels were similar relative to wild type. In contrast to $t t 3-1$, the three MBW mutants ttg1-1, tt8-3 and tt2-1 did not exhibit elevated kaempferol levels and all had reduced quercetin levels. Most strikingly, isorhamnetin levels were severely reduced in ttg1-1 (Fig. 4c, Additional file 7: Figure S10, Tables S12 and S13) but not in $t t 8-3$ and $t t 2-1$. This was not observed in $t t 8-3$ and $t t 2-1$ and therefore points to an independent role of TTG1 in regulating isorhamnetin.
In addition to TT2, four R2R3 MYB factors, PAP1 through 4, are known to regulate flavonoid biosynthesis together with TTG1. Overexpression of PAP1 in the activation tagging line pap1-D led to an accumulation of extracted hydrolysable cyanidin derivatives at the expense of quercetin, epicatechin and their respective derivatives (Fig. 4c,e). Non-extracted but hydrolysable derivatives of kaempferol, quercetin, cyanidin and epicatechin were significantly reduced in pap1-D seeds. None of the substances from our set was increased in the nonextracted hydrolysed samples. This suggests that less flavonoids were deposited in non-extracted substances as compared to the wild type. Possibly, PAP1 primarily regulates cyanidin modifying enzymes in seeds and thereby, when overexpressed, creates a sink situation affecting the seed's flavonoid composition.

The flavonoid composition of seeds from the mybRNAi line was similar to wild type. Few substances were significantly reduced as compared to wild type like quercetin and epicatechin. The latter could be explained by the transcriptional regulation of $A N R$ by $P A P(\mathrm{~s})$. This view is supported by the previous finding indicating that PAP4 overexpression in A. thaliana mesophyll protoplasts activates ANR [55]. Non-normalized and normalized results point to a slight downregulation of the pathway as no substance stood out when set relative to kaempferol. The results for pap1-D and the mybRNAi line suggest the (partially) redundant regulation of the flavonoid pathway.

In seedlings, HY5 is known to activate the expression of early and late biosynthesis genes by directly binding to the promoters of these genes [16]. hy5-215 seeds revealed various changes of the flavonoid composition In hydrolysed samples, kaempferol was significantly increased and most other flavonoids are reduced (Fig. 4c,e). This suggests a role of HY5 in the regulation of TT7 or downstream genes.

COP1 is predicted to affect the level of most substances in the pathway because it regulates the stability of relevant transcription factors like HY5 and PAP2 [19, 58]. As expected, the spectrum of extracted hydrolysable flavonoids accumulating in cop1-6 partially overlapped with that in pap1-D. In cop1-6 seeds, levels of cyanidin derivatives were high but not at the expense of other substances downstream of kaempferol as seen by normalization with kaempferol. In addition, we found several unexpected aspects of COP1 regulatory events influencing the flavonoid composition and deposition in seeds.

First, we revealed a role of COP1 in suppressing the accumulation of extracted quercetin- and isorhamnetinbased substances. One possibility is the mis-regulation of core enzymes in cop1-6. Alternatively, COP1 could suppress at least one quercetin and isorhamnetin 
modifying enzyme. Second, while high levels of cyanidin are present in the extracted hydrolysed samples, epicatechin levels remain unchanged as compared to wild type. A suppression of enzymes downstream of cyanidin and epicatechin is therefore unlikely. This points to a positive regulation of enzymes downstream of cyanidin which do not effect levels of epicatechin and downstream substances like cyanidin modifying (e.g. glycosylating) enzymes. Third, in contrast to an expected enhanced deposition of non-extracted condensed tannins or the extracted non-hydrolysed procyanidin B2 in cop1-6 seeds, we detected a significant decrease of substances released from the non-extracted fraction (taxifolin, pelargonidin, cyanidin and epicatechin).

\section{Adaptation of the method to seedlings}

Many studies analysing flavonoids have been conducted using seedlings. Protocols have been developed for specific needs ranging from photometric analysis to different types of metabolomics and other specific applications [37, 59, 60]. Here, we aimed to obtain fingerprints for shift detection from seedlings at high throughput. We used the extracted hydrolysis setup to adapt our method. Hydrolysed extracts from seedlings were subjected to LC-ESI-MSQTRAP(MRM) to select the optimal number of seedlings for screening purposes. Furthermore, we applied the experimental setup to selected mutants based on results from seeds.

Initially, a hydrolysis time of 60 min was employed as used in other studies $[33,52,53]$. This hydrolysis time proofed to be optimal, as none of the detected, quantifiable substances significantly increased when using 30 or $90 \mathrm{~min}$ (Fig. 5a, Additional file 8: Table S14). In addition, the major glycosylated flavonoids were absent after 60 min of hydrolysis (Additional file 8: Figure S11).

Detected responses for several substances approached a maximum at ten seedlings which turned out to be sufficient to detect most substances (Fig. 5b). Kaempferol responses were high and reached the maximum at five seedlings already. This required the analysis of diluted samples from five seedlings onwards for kaempferol detection (Fig. 5c). All substances found when using ten seedlings (taxifolin, naringenin, kaempferol, quercetin, isorhamnetin and cyanidin) were quantifiable in five and one seedling except for taxifolin which did not reach the limit of quantification in one seedling. In general, RSDs were reduced by the internal standard (Fig. 5b,c). Because ten seedlings are required to reach an RSD below $20 \%$ for all substances at quantifiable levels (Fig. 5d), we therefore recommend the use of ten seedlings.

Unexpected changes in the pathway which were revealed by fingerprinting of mutant seeds prompted us to analyse ttg1-1 seedlings and seedlings of light signalling mutants.
When comparing ttg1-1 to tt3-1 mutants, we again found evidence for a role of TTG1 in regulating TT7 since taxifolin levels were reduced in ttg1-1 when compared to $t t 3-1$. Relative to $t t 3-1$, isorhamnetin levels were drastically reduced and quercetin levels were slightly reduced in ttg1-1 mutants. This indicates that TTG1 regulates the accumulation of isorhamnetin in both, seeds and seedlings.

Differences in the flavonoid composition between seeds and seedlings were also found in mybRNAi lines. In hydrolysed extracts from mybRNAi seedlings, naringenin, taxifolin and cyanidin were significantly reduced while the flavonols were not affected. This suggests that $P A P$ genes regulate steps upstream of naringenin synthesis (e.g. $C H S$ and $C H I$ ). An additional regulation of $D F R$ and/or ANS is suggested by the finding that cyanidin levels were reduced, whereas quercetin levels remained unchanged. This is consistent with the previous finding that DFR expression can be activated by PAP1 and PAP2 [26]. An alternative explanation is the regulation of enzymes modifying flavonoids along the naringenin-taxifolincyanidin-branch.

In hy5-215, we found reduced levels of all substances downstream of naringenin. As kaempferol levels were increased in seeds, it is conceivable that HY5 acts at different steps in the flavonoid pathway in seeds and seedlings.

The extracts of pap1-D and cop1-6 had to be diluted 20 -fold to reach the linear range of the set of flavonoids. All $\mathrm{D}_{3}$-sakuranetin-normalized responses for cop1-6 in the dilutions were beyond the undiluted, normalized wild-type response. For pap1-D, similar as in seeds, cyanidin responses showed the strongest increase compared to the other detected substances and even reached similar levels as in cop1-6 mutants.

The levels of all analysed substances upstream of cyanidin were lower in pap1-D compared to those in cop1-6. We therefore conclude that COP1 is a master regulator of the flavonoid biosynthesis pathway in seedlings but not of flavonoid deposition in seeds.

\section{Discussion}

The qualitative and quantitative analysis of flavonoids in plants is well established. Photometric methods enable a quick estimation of anthocyanidin contents [33, 60] or, in combination with LC and UV detectors, an assessment of their composition (e.g. [33, 61]). A more detailed analysis is done by LC-MS using e.g. MRM, TOF detectors, MSMS or pseudoMS ${ }^{3}$ [42-46]. Depending on the specific questions either the extracted fractions or the non-extracted hydrolysed fraction are used. Typically, methods compromise in many respects to adapt for the specific goal. The method established here, is optimized for situations in which the whole flavonoid 


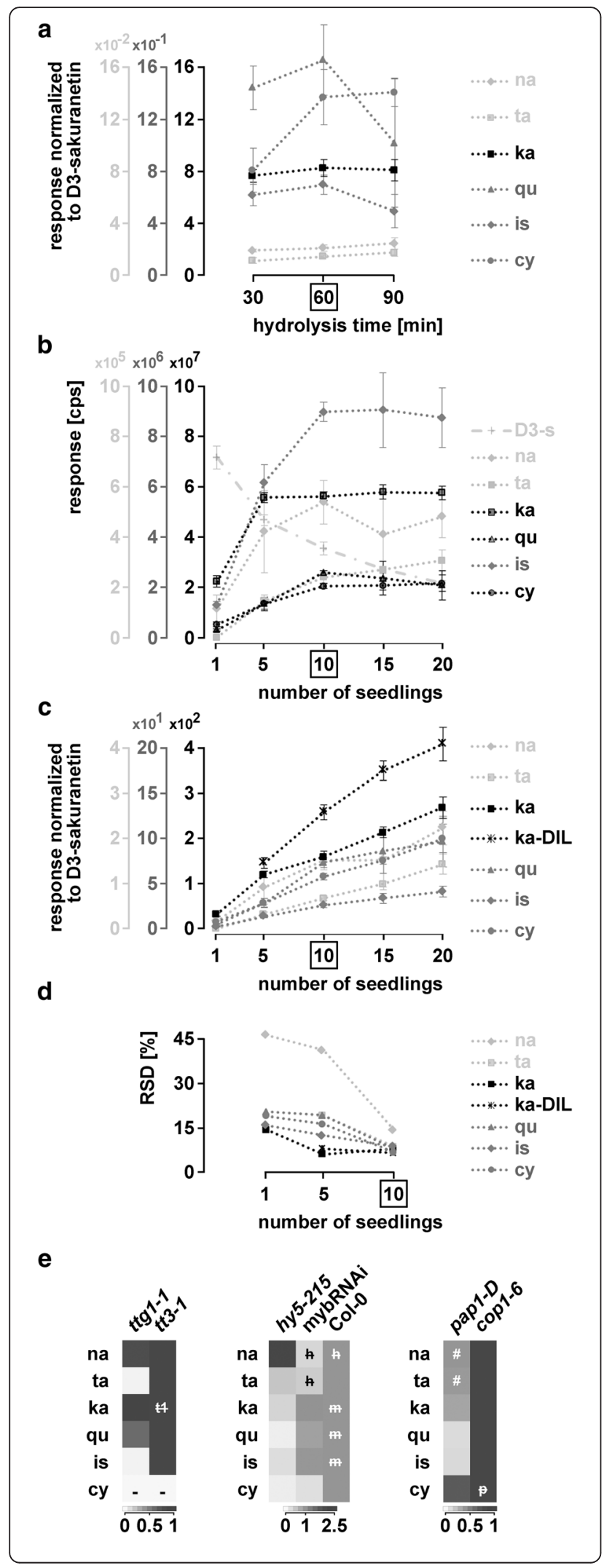

Fig. 5 Adaptation of the method to seedlings. Analysis of seedlings by LC-ESI-MS-QTRAP(MRM). a $\mathrm{D}_{3}$-sakuranetin-normalized responses of flavonoids tested for different times of hydrolysis. b-d Selection of the optimal number of seedlings for the set of analysed flavonoids. b Responses of the different flavonoids tested, including $D_{3}$-sakuranetin, for $1,5,10,15$ and 20 seedlings. cps: counts per second. $\mathrm{c}_{3^{-}}$ sakuranetin-normalized responses from (b) and diluted samples for kaempferol. Please note that only quantifiable amounts are shown (Additional file 8: Table S14) d) RSD in percent for 1,5 and 10 seedlings from (c). e Mutants. Heatmaps showing $\log _{2}$ transformed $D_{3}$-sakuranetin-normalized responses $(n=3)$ relative to $t t 3-1$, Col-0 and cop 1-6. Statistics and mean normalized responses: Additional file 8: Table S14. Boxed: optimal number of seedlings according to these experiments. Error bars = STDEV. \# LOD but not LOQ passed. -: LOD not passed. $h, m, p$ and $t 1$ : no significant difference to the respective mutant. h: hy5-215, m: mybRNAi, p: pap 1-D, t1: ttg1-1. ka-DIL: kaempferol-diluted

pathway needs to be quantitatively monitored in many samples and with limited biological material. Three aspects were relevant for the optimization. First, we determined the shortest hydrolysis time by considering two criteria: the complete removal of glycosylated modifications and the minimal conversions of flavonoids into others. The latter is important to avoid false signals leading to a misinterpretation of alterations in the pathway. Second, the method is suitable to detect most key flavonoids in only one seed or seedling. Using this fingerprint, it is possible to compare alterations in the flavonoid pathway in many biological samples. Third, we combine three types of samples, the extracted nonhydrolysed, the extracted hydrolysed and the nonextracted hydrolysed samples. This combination proofed to be very useful to decide whether individual flavonoids have been released from extracted or non-extracted derivatives. In our study it was particularly helpful to recognize a role of COP1 in the regulation of enzymes modifying quercetin, isorhamnetin and cyanidin.

Our analysis of the flavonoid pathway in mutants revealed several interesting new findings. It is well accepted that DFR is regulated by TTG1, the bHLH protein TT8 and the R2R3MYB protein TT2 possibly in a TTG1-MBW complex [27, 57]. One would therefore expect that ttg1-1 mutants show a similar flavonoid composition and levels as $t t 3-1, t t 8-3$ and $t t 2-1$ mutants. This is clearly not the case. On the one hand, ttg1-1 mutants do not exhibit the expected increase in isorhamnetin levels but rather a reduction. On the other hand, $t t 8$ 3 and $t$ t2-1 mutants differ from $t$ tg $1-1$ as they show wild-type levels of isorhamnetin. Moreover, $\operatorname{ttg} 1-1, t t 8-3$ and $t t 2-1$ exhibit decreased quercetin levels which is not seen in $t t 3-1$. These data suggest two regulatory features of TTG1. First, the decreased quercetin levels suggest that TTG1, TT8 and TT2 are involved in earlier steps of the pathway or quercetin modification. One likely explanation is its regulation of TT7 as reported before 
[28]. The different compositions in $t t 8-3$ and $t t 2-1 \mathrm{mu}-$ tants indicate that TTG1 also exerts this function independently of TT8 and TT2. Possible targets are OMT or isorhamnetin modifying enzymes.

The role of TTG1 in regulating isorhamnetin is also found in seedlings suggesting that TTG1 regulates OMT or the isorhamnetin modifying enzyme in both tissues in the same manner. By contrast we found a tissue-specific regulation of the flavonoid pathway in light signalling mutants, in particular by COP1.

While COP1 has little impact on the flavonoid pathway in seeds, it is the master regulator in seedlings. This is indicated by the findings that for all detected substances in seedlings, cop1-6 mutants released the highest levels.

Differential regulation of the flavonoid pathway in seeds and seedlings were also observed in mutants of transcription factors downstream of COP1. Similar to cop1-6 mutants, pap1-D seedlings exhibited highly increased cyanidin levels relative to the other flavonoids. In mybRNAi lines naringenin, taxifolin and cyanidin levels are reduced in seedlings but not in seeds. Interestingly, HY5 is equally important in seeds and seedlings but acts at different levels of the pathway. In seeds, kaempferol accumulates in the hy5-215 mutant but is reduced in seedlings, which suggests that HY5 regulates steps upstream of TT7 in seedlings, whereas in seeds, TT7 or downstream enzymes are controlled. Together, our data support a dependence on the developmental stage for COP1 function: while the flavonoid composition of seeds is only moderately dependent on the COP1-dependent light signalling pathway, COP1 is a master regulator of the pathway in seedlings.

\section{Conclusions}

In this work, we developed a method for the analysis of the flavonoid biosynthesis pathway optimized for purposes for which quantitative comparison with limited plant material is important. In proof of principle experiments the method revealed a differential regulation of kaempferol by HY5 in seedlings versus seeds and provided evidence for the role of COP1 as a master regulator of the pathway in seedlings but not for flavonoid deposition in seeds. This method will be helpful in genetic studies and in particular for the analysis of natural variation in different ecotypes or genetic mutants.

\section{Methods}

\section{Plant material and growth condition}

All mutants used in this study have been described before $[7,28,57,62-73]$. The following mutants can be obtained from the Nottingham Arabidopsis Stock Centre (NASC): $t t 2-1$ (N83), $t t 3-1$ (N84), tt4-11 (N2105573), tt7-1 (N88), tt8-3 (N891), pap1-D (N3884), cop1-6
(N69041). Mutants and primers for genotyping are listed in Additional file 9: Tables S15 and S16. For designing dCAPS primers, dCAPSfinder 2.0 was used [74].

All $A$. thaliana ripened seeds originated from plants grown at $21^{\circ} \mathrm{C}$ in $16 / 8 \mathrm{~h}$ light $\left(90-120 \mu \mathrm{mol}^{*} \mathrm{~m}^{-2} \mathrm{~s}^{-1}\right.$, LUMILUX Cool white L58W/840, Osram, http:// www.osram.de). For seedling analysis, seeds were sown on solid MS plates (1\% sucrose), kept in darkness at $4^{\circ} \mathrm{C}$ for 2.5 days prior to transfer to white light $\left(4 \mathrm{~d}, 21^{\circ} \mathrm{C}, 40\right.$ $\mu \mathrm{mol}^{*} \mathrm{~m}^{-2} \mathrm{~s}^{-1}$, FLUORA L58W/77, Osram, http:// www.osram.de).

\section{Chemicals, external standards}

$\mathrm{MeOH}$ (CHROMASOLV, Fluka, http://www.sigmaaldrich.com), formic acid (FA) and $\mathrm{HCl}$ conc. (both NORMAPUR, AnalaR, www.vwr.com) were used at LC-MS grade. The external standards (Sigma-Aldrich (https:// www.sigmaaldrich.com), Roth (https://www.carlroth.com)) are listed in Additional file 1: Table S1. For quality control of LC-MS analysis all 12 external standards were mixed $(100 \mathrm{nM})$ with $400 \mathrm{nM} \mathrm{D} \mathrm{D}_{3}$-quercetin and $5 \mathrm{nM}$ $\mathrm{D}_{3}$-sakuranetin. (For experiments with external standards: see Additional file 9: Methods S2.)

\section{Internal standards synthesis}

To synthesize $\mathrm{D}_{3}$-Quercetin, we followed a modified three-step procedure using a $\mathrm{BF}_{3} \mathrm{THF}$ solution instead of $\mathrm{BF}_{3}$ gas $[75,76]$. The enrichment was analysed by ESIMS using a Thermo Exactive Orbitrap MS (Thermo Fisher, http://www.thermoscientific.com). $\mathrm{D}_{3}$-sakuranetin was synthesised by methylation of naringenin as shown before [77] using deuterated methyl iodide under basic conditions. All methods for internal standard synthesis are documented in Additional file 3: Methods S1.

\section{Flavonoid extraction}

Protocols are described as derived after optimizing the $\mathrm{MeOH}$ concentration for seeds, seed(ling) number and hydrolysis time for seed(ling)s. In all experiments, quintuplicates are analysed if not stated otherwise.

Ten seeds were homogenized in $100 \mu \mathrm{l} \mathrm{MeOH}$ :$\mathrm{H}_{2} \mathrm{O}$ :FA (50:49:1), spiked with $10 \mu \mathrm{D}_{3}$-quercetin (100 $\mu \mathrm{M}$ ) using a TISSUELYSER (Qiagen, https://www.qiagen.com) with three glass beads (BIOSPEC, \#11079125/ N032.1, Roth, www.carlroth.com) for $3 \mathrm{~min}$ at $30 \mathrm{~Hz}$. Upon centrifugation (14000 rpm, $\left.5 \mathrm{~min}, 4^{\circ} \mathrm{C}\right)$, the pellet was re-extracted in $200 \mu \mathrm{l}$ of extraction buffer $\left(4^{\circ} \mathrm{C}\right.$ overnight) followed by centrifugation. $60 \mu \mathrm{l}$ of combined supernatants were mixed with $440 \mu \mathrm{l} \mathrm{MeOH}$ (extracted non-hydrolysed sample). $120 \mu \mathrm{l}$ of the supernatants and $380 \mu \mathrm{l} \mathrm{MeOH}: H C l c o n c . ~(95: 5)$ were hydrolysed $\left(99{ }^{\circ} \mathrm{C}\right.$, $20 \mathrm{~min})$ and diluted 1:1 with $\mathrm{D}_{3}$-sakuranetin(200 $\mathrm{nM}): \mathrm{MeOH}$ (1:20) (extracted hydrolysed sample). $\mathrm{D}_{3^{-}}$ sakuranetin served in this sample to monitor fluctuation 
of the machine. For non-extracted proanthocyanidins, the pellet was hydrolysed $\left(99^{\circ} \mathrm{C}, 10 \mathrm{~min}\right)$ with $210 \mu \mathrm{l}$ of $\mathrm{D}_{3}$ sakuranetin(200nM):MeOH:HClconc (1:19:1). Supernatants were diluted 1:1 with $\mathrm{MeOH}$ following two rounds of centrifugation (non-extracted hydrolysed sample).

$10 \mathrm{~N}_{2}$ liq-frozen seedlings with $210 \mu \mathrm{l}$ of $\mathrm{D}_{3}$-sakuranetin(210nM): $\mathrm{MeOH}$ (1:20) were homogenized as above but with $22 \mathrm{~Hz}$, incubated in darkness $\left(4^{\circ} \mathrm{C}\right.$ overnight) and centrifuged (14000rpm, 12min). $100 \mu \mathrm{l}$ of the supernatant were hydrolysed $\left(99^{\circ} \mathrm{C}, 60 \mathrm{~min}\right)$, centrifuged and $60 \mu \mathrm{l}$ supernatant were diluted 1:1 with $\mathrm{MeOH}$. For 10 Col-0 and mutant seedlings, these extracts were further diluted 1:6 with $\mathrm{MeOH}$ to analyse kaempferol in the linear range (Fig. 5 c,d).

\section{LC-MS}

A KINETEX $2.6 \mu \mathrm{m}$ C18 $100 \AA$ (4.6 mm x $50 \mathrm{~mm}) \mathrm{C} 18$ column (Phenomenex, http://www.phenomenex.com) was used for all samples. For quantification via LC-MS/MS in MRM mode (=LC-ESI-MS-QTRAP(MRM)) an Agilent 1260 HPLC (http://www.agilent.com/home) coupled to a QTRAP 5500 mass spectrometer (ABSCIEX, http://sciex.com) were used; for identification (AutoMSMS and pseudoMS $^{3}$ experiments), a DIONEX 3000 RSL UPLC (Thermo Fisher, http://www.dionex.com) system was coupled to a MAXIS 4G (Bruker Daltonic, www.bruker.com) (=LC-ESI-MS-QTOF(AutoMSMS or pseudoMS $\left.{ }^{3}\right)$ ). For details on LC-MS see Additional file 9: Methods S2.

\section{Detection of glycosylated flavonoids and identification of their aglycons}

Extracted non-hydrolysed samples were injected twice and subjected once to LC-ESI-MS-QTOF(AutoMSMS) and once to LC-ESI-MS-QTOF(pseudoMS ${ }^{3}$ ). The Auto MSMS run revealed glycosylated flavonoids, the pseudoMS $^{3}$ run identified the specific flavonoid via MS/MS fragmentation pattern as compared to the respective external standards. Both contribute through neutral loss analysis to identify the type of glycosylation (here: hexose or deoxy-hexose, for further details and reference $m$ / $z$ values see Additional file 2: Tables S3-S6).

\section{General data analysis, statistics, and specific visualization}

Using non-smoothed data, the limit of detection (LOD) was defined at a signal-to-noise ratio $(\mathrm{S} / \mathrm{N})$ of 3 and the limit of quantification (LOQ) at a $\mathrm{S} / \mathrm{N}$ of 10 . LOD or LOQ was assigned for a mean when more than half of the replicates passed the respective threshold. All raw LC-ESI-MS-QTRAP data were analysed using Analyst 1.6 (ABSCIEX, http://sciex.com). Integrated peak areas from the inbuilt Analyst Quantitation Wizard were manually corrected. LC-ESI-MS-QTOF raw data were mass corrected with sodium formate clusters using HPC with Compass AutomationEngine 4.1. Subsequent analysis was performed with Compass DataAnalysis Version 4.1 SP5.

Statistic analysis was done using R 3.0.2 (Shapiro test, Welch test, Wilcoxon rank sum test) [78-81]. Heatmaps were generated for $\log _{10}$ transformed values with heatmap.2 in $\mathrm{R}$ 3.0.1 using gplots and RColorBrewer [82, 83]. All other heatmaps were generated with the BAR HeatMapper Plus Tool (http://bar.utoronto.ca/ntools/ cgi-bin/ntools_heatmapper_plus.cgi) and changes to greyscale.

\section{Additional files}

Additional file 1: Table S1. Optimizing for high throughput. List of external standards; and Table S2. Transition parameters. (PDF 381 kb)

Additional file 2: Figure S1. Concept of hydrolysis. Reduced complexity of extracts after hydrolysis; Table S3. Calculated m/z values for detection of glycosylated flavonoids; Table S4. Glycosylated flavonoids in extracted non-hydrolysed samples from A. thaliana seeds; Table S5. Glycosylated flavonoids in extracted non-hydrolysed samples from $A$. thaliana seedlings; and Table S6. Calculated m/z values for selected substances. (PDF $1704 \mathrm{~kb}$ )

Additional file 3: Figure S2. External and Internal standards. EICs of external standards; Figure S3. Linearity and EICs of deuterated, internal standards; Table S7. Stability of standards over time and in dependence of acid, Table S8. Precision and linearity of the selected external standards; and Methods S1. Internal standard synthesis. (PDF 1682 kb)

Additional file 4: Figure S4. Extraction and hydrolysis time. Selection of the methanol concentration; Figure S5. Non-adjusted responses from Figure S4; Figure S6. Seed extracts treated with different hydrolysis times; Table S9. Data for Figures S4, S5; and Table S10. Data for Fig. 2 and Figure S6. (PDF $1210 \mathrm{~kb}$ )

Additional file 5: Figure S7. Effect of hydrolysis on reference substances. Effect of hydrolysis on analysed flavonoids; and Figure S8. Release of pelargonidin from the internal standard and cyanidin from pelargonidin. (PDF $493 \mathrm{~kb}$ )

Additional file 6: Figure S9. Minimizing the number of seeds. Reduction of seed material down to one seed - repetition; and Table S11. Data for Fig. 4a,b. (PDF 356 kb)

Additional file 7: Figure S10. Proof of principle: revealing shifts in the pathway. Application (seeds of mutants) - normalized responses; Table S12. Data for Fig. 4c-e.; and Table S13. Data for Fig. 4c,d - significance. (PDF $731 \mathrm{~kb}$ )

Additional file 8: Figure S11. Adaptation of the method to seedlings. Hydrolysis of main glycosylated flavonoids from seedling extracts; and Table S14. Data for Fig. 6. (PDF 445 kb)

Additional file 9: Table S15. Mutants and primers. Mutants and insertion lines used in this study; Table S16. Primer; and Methods S2. (PDF $431 \mathrm{~kb}$ )

\section{Acknowledgement}

The authors acknowledge the significant contribution provided by the Biocenter-MS platform at the University of Cologne. For making available seeds we like to thank: M. Koornneef (tt2-1, tt7-1), B. Weisshaar and I. Appelhagen (tt4-11), A. Lloyd (mybRNAi), ABRC (pap1-D). We thank M. Schäfer for discussion on deuterated substances and required analytics, E. von Elert for providing access to his Thermo Exactive Orbitrap MS and Thomas Sadler for help with this device, B. Welter for technical assistance and S. Metzger for feedback on basic LC-MS data analysis.

\section{Funding}

This work was supported by a Grant from the Deutsche Forschungsgemeinschaft SFB 680 to M.H. and by the Cluster of Excellence on Plant Sciences to M.H., U.H., M.B. (CEPLAS; EXC 1028). B.J. was supported by the IMPRS Graduate School. X.Y. was supported by the IMPRS Graduate School and the University of Cologne. 


\section{Availability of data and material}

All supporting data can be found within the manuscript and its additional files. The raw data are available from the corresponding author on reasonable request. Both internal standards were synthetized by A.O.T./H.-G.S. and handed over into the responsibility of the Biocenter-MS platform, University of Cologne. Details on the synthesis and characterisation of both internal standards can be found within the manuscript and in the additional files (Additional file 3.pdf).

\section{Authors' contributions}

$\mathrm{MB}, \mathrm{UH}, \mathrm{MH}$, AS designed the project; AS did the project administration; $\mathrm{MH}$, AS did the writing; MKU optimized LC-MS setups; CL, MKU applied LC-MS setups; CL, MKU, AS characterized standards; AOT, H-GS developed methodology, performed and validated internal standards synthesis; $X Y, A S$, UH optimized seedling extraction and methodology; BJ, AS optimized seed extraction and methodology; AS did all integration, analysed and validated all data; BJ validated data and did statistic analysis using $\mathrm{R}$; all interpreted data and did proof reading. All authors have read and approved the manuscript.

\section{Competing interests}

The authors declare that they have no competing interests.

\section{Consent for publication}

Not applicable.

\section{Ethics approval and consent to participate}

Not applicable.

\section{Author details}

'Botanical Institute and Cluster of Excellence on Plant Sciences (CEPLAS), University of Cologne, Cologne Biocenter, Zülpicher Str. 47b, 50674 Cologne, Germany. ${ }^{2}$ Department of Chemistry, University of Cologne, Greinstr. 4, 50939 Cologne, Germany.

\section{Received: 27 May 2016 Accepted: 23 August 2016}

\section{Published online: 01 September 2016}

\section{References}

1. Corder R, Mullen W, Khan NQ, Marks SC, Wood EG, Carrier MJ, et al. Red wine procyanidins and vascular health. Nature. 2006;444:566.

2. Takahashi A, Takeda K, Ohnishi T. Light-induced anthocyanin reduces the extent of damage to DNA in UV-irradiated centaurea cyanus cells in culture. Plant Cell Physiol. 1991;32:541-7.

3. Winkel-Shirley B. Flavonoid biosynthesis. A colorful model for genetics, biochemistry, cell biology, and biotechnology. Plant Physiol. 2001;126:485-93.

4. Hichri I, Barrieu F, Bogs J, Kappel C, Delrot S, Lauvergeat V. Recent advances in the transcriptional regulation of the flavonoid biosynthetic pathway. J Exp Bot. 2011;62:2465-83.

5. Pojer E, Mattivi F, Johnson D, Stockley CS. The case for anthocyanin consumption to promote human health: a review. Compr Rev Food Sci Food Saf. 2013;12:483-508.

6. Chalker-Scott L. Environmental significance of anthocyanins in plant stress responses. Photochem Photobiol. 1999;70:1-9.

7. Koornneef M. The complex syndrome of ttg mutants. Arabidopsis Inf Serv. 1981;18:45-51

8. Koornneef M. Mutations affecting the testa colour in Arabidopsis Arabidopsis Inf Serv. 1990;27:1-4.

9. Lepiniec L, Debeaujon I, Routaboul JM, Baudry A, Pourcel L, Nesi N, et al. Genetics and biochemistry of seed flavonoids. Annu Rev Plant Biol. 2006;57:405-30.

10. Tanner G. Condensed tannins. In: Davies KM editor. Plant Pigments and their Manipulation. Oxford: Blackwell Publishing Ltd; 2004. p. 150-84.

11. Saito K, Yonekura-Sakakibara K, Nakabayashi R, Higashi Y, Yamazaki M, Tohge $T$, et al. The flavonoid biosynthetic pathway in Arabidopsis: structural and genetic diversity. Plant Physiol Biochem. 2013;72:21-34.

12. Yonekura-Sakakibara K, Nakayama T, Yamazaki M, Saito K. Modification and Stabilization of Anthocyanins. In: Winefield C, Davies K, editors. Gould K editor. Anthocyanins: Springer New York; 2009. p. 169-90.

13. Liu J, Osbourn A, Ma P. MYB Transcription factors as regulators of phenylpropanoid metabolism in plants. Mol Plant. 2015;8:689-708.
14. Broun P. Transcriptional control of flavonoid biosynthesis: a complex network of conserved regulators involved in multiple aspects of differentiation in Arabidopsis. Curr Opin Plant Biol. 2005;8:272-9.

15. Petroni $\mathrm{K}$, Tonelli $\mathrm{C}$. Recent advances on the regulation of anthocyanin synthesis in reproductive organs. Plant Sci. 2011;181:219-29.

16. Shin J, Park E, Choi G. PIF3 regulates anthocyanin biosynthesis in an HY5dependent manner with both factors directly binding anthocyanin biosynthetic gene promoters in Arabidopsis. Plant J. 2007;49:981-94.

17. Zhu D, Maier A, Lee JH, Laubinger S, Saijo Y, Wang H, et al. Biochemical characterization of Arabidopsis complexes containing CONSTITUTIVELY PHOTOMORPHOGENIC1 and SUPPRESSOR OF PHYA proteins in light control of plant development. Plant Cell. 2008;20:2307-23.

18. Osterlund MT, Hardtke CS, Wei N, Deng XW. Targeted destabilization of HY5 during light-regulated development of Arabidopsis. Nature. 2000;405:462-6.

19. Maier A, Schrader A, Kokkelink L, Falke C, Welter B, Iniesto E, et al. Light and the E3 ubiquitin ligase COP1/SPA control the protein stability of the MYB transcription factors PAP1 and PAP2 involved in anthocyanin accumulation in Arabidopsis. Plant J. 2013;74:638-51.

20. Cominelli E, Gusmaroli G, Allegra D, Galbiati M, Wade HK, Jenkins Gl, et al. Expression analysis of anthocyanin regulatory genes in response to different light qualities in Arabidopsis thaliana. J Plant Physiol. 2008;165:886-94.

21. Ma L, Gao Y, Qu L, Chen Z, Li J, Zhao H, et al. Genomic evidence for COP1 as a repressor of light-regulated gene expression and development in Arabidopsis. Plant Cell. 2002;14:2383-98.

22. von Arnim AG, Deng XW. Light inactivation of Arabidopsis photomorphogenic repressor COP1 involves a cell-specific regulation of its nucleocytoplasmic partitioning. Cell. 1994;79:1035-45.

23. Pacin M, Legris M, Casal JJ. Rapid decline in nuclear costitutive photomorphogenesis1 abundance anticipates the stabilization of its target elongated hypocotyl5 in the light. Plant Physiol. 2014;164:1134-8.

24. Yu JW, Rubio V, Lee NY, Bai S, Lee SY, Kim SS, et al. COP1 and ELF3 control circadian function and photoperiodic flowering by regulating $\mathrm{Gl}$ stability. Mol Cell. 2008;32:617-30

25. Deng XW, Caspar T, Quail PH. cop1: a regulatory locus involved in lightcontrolled development and gene expression in Arabidopsis. Genes Dev. 1991;5:1172-82.

26. Zimmermann IM, Heim MA, Weisshaar B, Uhrig JF. Comprehensive identification of Arabidopsis thaliana MYB transcription factors interacting with R/B-like BHLH proteins. Plant J. 2004;40:22-34.

27. Ramsay NA, Glover BJ. MYB-bHLH-WD40 protein complex and the evolution of cellular diversity. Trends Plant Sci. 2005;10:63-70.

28. Gonzalez A, Zhao M, Leavitt JM, Lloyd AM. Regulation of the anthocyanin biosynthetic pathway by the $T \mathrm{G} 1 / \mathrm{bHLH} / \mathrm{Myb}$ transcriptional complex in Arabidopsis seedlings. Plant J. 2008;53:814-27.

29. Debeaujon I, Nesi N, Perez P, Devic M, Grandjean O, Caboche M, et al. Proanthocyanidin-accumulating cells in Arabidopsis testa: regulation of differentiation and role in seed development. Plant Cell. 2003;15:2514-31.

30. Baudry A, Heim MA, Dubreucq B, Caboche M, Weisshaar B, Lepiniec L. TT2, TT8, and TTG1 synergistically specify the expression of BANYULS and proanthocyanidin biosynthesis in Arabidopsis thaliana. Plant J. 2004;39:366-80.

31. Yonekura-Sakakibara K, Tohge T, Matsuda F, Nakabayashi R, Takayama H, Niida R, et al. Comprehensive flavonol profiling and transcriptome coexpression analysis leading to decoding gene-metabolite correlations in Arabidopsis. Plant Cell. 2008;20:2160-76.

32. Tohge T, Nishiyama Y, Hirai MY, Yano M, Nakajima J, Awazuhara M, et al. Functional genomics by integrated analysis of metabolome and transcriptome of Arabidopsis plants over-expressing an MYB transcription factor. Plant J. 2005:42:218-35.

33. Routaboul JM, Kerhoas L, Debeaujon I, Pourcel L, Caboche M, Einhorn J, et al. Flavonoid diversity and biosynthesis in seed of Arabidopsis thaliana. Planta. 2006;224:96-107.

34. Kerhoas L, Aouak D, Cingoz A, Routaboul JM, Lepiniec L, Einhorn J, et al. Structural characterization of the major flavonoid glycosides from Arabidopsis thaliana seeds. J Agric Food Chem. 2006:54:6603-12.

35. Vogt T. Phenylpropanoid biosynthesis. Mol Plant. 2010;3:2-20

36. Wolfender JL, Marti G, Thomas A, Bertrand S. Current approaches and challenges for the metabolite profiling of complex natural extracts. J Chromatogr A. 2015:1382:136-64.

37. Saito K, Matsuda F. Metabolomics for functional genomics, systems biology, and biotechnology. Annu Rev Plant Biol. 2010;61:463-89.

38. Burwell RL. The cleavage of ethers. Chem Rev. 1954;54:615-85. 
39. Porter LJ, Hrstich LN, Chan BG. The conversion of procyanidins and prodelphinidins to cyanidin and delphinidin. Phytochemistry. 1985;25:223-30

40. Peng Z, Hayasaka Y, lland PG, Sefton M, Hoj P, Waters EJ. Quantitative analysis of polymeric procyanidins (Tannins) from grape (Vitis vinifera) seeds by reverse phase high-performance liquid chromatography. J Agric Food Chem. 2001;49:26-31.

41. Forsyth WG, Roberts JB. Cacao polyphenolic substances. 5. The structure of cacao 'leucocyanidin 1. Biochem J. 1960;74:374-8.

42. Wolfender JL. HPLC in natural product analysis: the detection issue. Planta Med. 2009;75:719-34.

43. Bottcher C, von Roepenack-Lahaye E, Schmidt J, Schmotz C, Neumann S, Scheel $D$, et al. Metabolome analysis of biosynthetic mutants reveals a diversity of metabolic changes and allows identification of a large number of new compounds in Arabidopsis. Plant Physiol. 2008;147:2107-20.

44. Marczak L, Kachlicki P, Kozniewski P, Skirycz A, Krajewski P, Stobiecki M. Matrix-assisted laser desorption/ionization time-of-flight mass spectrometry monitoring of anthocyanins in extracts from Arabidopsis thaliana leaves. Rapid Commun Mass Spectrom. 2008;22:3949-56.

45. Konishi Y, Kiyota T, Draghici C, Gao JM, Yeboah F, Acoca S, et al. Molecular formula analysis by an MS/MS/MS technique to expedite dereplication of natural products. Anal Chem. 2007;79:1187-97.

46. Stobiecki M, Skirycz A, Kerhoas L, Kachlicki P, Muth D, Einhorn J, et al. Profiling of phenolic glycosidic conjugates in leaves of Arabidopsis thaliana using LC/MS. Metabolomics. 2006;2:197-219.

47. Tohge T, Fernie AR. Combining genetic diversity, informatics and metabolomics to facilitate annotation of plant gene function. Nat Protoc. 2010:5:1210-27.

48. Goossens A, Hakkinen ST, Laakso I, Seppanen-Laakso T, Biondi S, De Sutter $V$, et al. A functional genomics approach toward the understanding of secondary metabolism in plant cells. Proc Natl Acad Sci U S A. 2003;100:8595-600.

49. Saito K, Hirai MY, Yonekura-Sakakibara K. Decoding genes with coexpression networks and metabolomics - 'majority report by precogs'. Trends Plant Sci. 2008;13:36-43.

50. Pelletier MK, Burbulis IE, Winkel-Shirley B. Disruption of specific flavonoid genes enhances the accumulation of flavonoid enzymes and end-products in Arabidopsis seedlings. Plant Mol Biol. 1999;40:45-54.

51. Martens S, Preuss A, Matern U. Multifunctional flavonoid dioxygenases: Flavonol and anthocyanin biosynthesis in Arabidopsis thaliana L. Phytochemistry. 2010;71:1040-9.

52. Tohge T, Matsui K, Ohme-Takagi M, Yamazaki M, Saito K. Enhanced radical scavenging activity of genetically modified Arabidopsis seeds. Biotechnol Lett. 2005;27:297-303.

53. Kitamura S, Matsuda F, Tohge T, Yonekura-Sakakibara K, Yamazaki M, Saito K, et al. Metabolic profiling and cytological analysis of proanthocyanidins in immature seeds of Arabidopsis thaliana flavonoid accumulation mutants. Plant J. 2010;62:549-59.

54. Matsuda F, Yonekura-Sakakibara K, Niida R, Kuromori T, Shinozaki K, Saito K. MS/MS spectral tag-based annotation of non-targeted profile of plant secondary metabolites. Plant J. 2009:57:555-77.

55. Heppel SC, Jaffe FW, Takos AM, Schellmann S, Rausch T, Walker AR, et al. Identification of key amino acids for the evolution of promoter target specificity of anthocyanin and proanthocyanidin regulating MYB factors. Plant Mol Biol. 2013;82:457-71.

56. Stracke R, Jahns O, Keck M, Tohge T, Niehaus K, Fernie AR, et al. Analysis of PRODUCTION OF FLAVONOL GLYCOSIDES-dependent flavonol glycoside accumulation in Arabidopsis thaliana plants reveals MYB11-, MYB12- and MYB111-independent flavonol glycoside accumulation. New Phytol. 2010;188:985-1000.

57. Nesi N, Debeaujon I, Jond C, Pelletier G, Caboche M, Lepiniec L. The TT8 gene encodes a basic helix-loop-helix domain protein required for expression of DFR and BAN genes in Arabidopsis siliques. Plant Cell. 2000;12:1863-78.

58. Hoecker U. Regulated proteolysis in light signaling. Curr Opin Plant Biol. 2005:8:469-76.

59. Kueger S, Steinhauser D, Willmitzer L, Giavalisco P. High-resolution plant metabolomics: from mass spectral features to metabolites and from wholecell analysis to subcellular metabolite distributions. Plant J. 2012;70:39-50.

60. Lange $\mathrm{H}$, Shropshire J, Mohr H. An analysis of phytochrome-mediated anthocyanin synthesis. Plant Physiol. 1971;47:649-55.
61. Bowerman PA, Ramirez MV, Price MB, Helm RF, Winkel BS. Analysis of T-DNA alleles of flavonoid biosynthesis genes in Arabidopsis ecotype Columbia. BMC Res Notes. 2012;5:485

62. Nesi N, Jond C, Debeaujon I, Caboche M, Lepiniec L. The Arabidopsis TT2 gene encodes an R2R3 MYB domain protein that acts as a key determinant for proanthocyanidin accumulation in developing seed. Plant Cell. 2001;13:2099-114.

63. Alonso JM, Stepanova AN, Leisse TJ, Kim CJ, Chen H, Shinn P, et al. Genome-wide insertional mutagenesis of Arabidopsis thaliana. Science. 2003;301:653-7.

64. Appelhagen I, Thiedig K, Nordholt N, Schmidt N, Huep G, Sagasser M, et al. Update on transparent testa mutants from Arabidopsis thaliana: characterisation of new alleles from an isogenic collection. Planta. 2014;240:955-70.

65. Buer CS, Muday GK, Djordjevic MA. Flavonoids are differentially taken up and transported long distances in Arabidopsis. Plant Physiol. 2007;145:478-90.

66. Oyama T, Shimura Y, Okada K. The Arabidopsis HY5 gene encodes a bZIP protein that regulates stimulus-induced development of root and hypocotyl. Genes Dev. 1997;11:2983-95.

67. Borevitz JO, Xia Y, Blount J, Dixon RA, Lamb C. Activation tagging identifies a conserved MYB regulator of phenylpropanoid biosynthesis. Plant Cell. 2000;12:2383-94.

68. McNellis TW, von Arnim AG, Araki T, Komeda Y, Misera S, Deng XW. Genetic and molecular analysis of an allelic series of cop1 mutants suggests functional roles for the multiple protein domains. Plant Cell. 1994;6:487-500.

69. Schoenbohm C, Martens S, Eder C, Forkmann G, Weisshaar B. Identification of the Arabidopsis thaliana flavonoid 3'-hydroxylase gene and functional expression of the encoded P450 enzyme. Biol Chem. 2000;381:749-53.

70. Koornneef M, Luiten W, Vlaming P, Schram AW. A gene controlling flavonoid-3'-hydroxylation in Arabidopsis. Arabidopsis Inf Serv. 1982;19:113-5.

71. Shirley BW, Hanley S, Goodman HM. Effects of ionizing radiation on a plant genome: analysis of two Arabidopsis transparent testa mutations. Plant Cell. 1992:4:333-47.

72. Koornneef M, Dellaert LWM, van der Veen JH. EMS- and relation-induced mutation frequencies at individual loci in Arabidopsis thaliana (L.) Heynh. Mutat Res. 1982;93:109-23.

73. Scholl RL, May ST, Ware DH. Seed and molecular resources for Arabidopsis. Plant Physiol. 2000;124:1477-80.

74. Neff MM, Turk E, Kalishman M. Web-based primer design for single nucleotide polymorphism analysis. Trends Genet. 2002;18:613-5.

75. Wahala K, Rasku S. Synthesis of D-4-genistein, a stable deutero labeled isoflavone, by a predeuteration - Selective dedeuteration approach. Tetrahedron Lett. 1997;38:7287-90.

76. Rasku S, Wahala K. Synthesis of deuterium labeled polyhydroxy flavones and 3-flavonols. Tetrahedron. 2000;56:913-6.

77. Oyama K, Kondo T. Total synthesis of flavocommelin, a component of the blue supramolecular pigment from Commelina communis, on the basis of direct 6-C-glycosylation of flavan. J Org Chem. 2004;69:5240-6.

78. R Core Team R. A Language and Environment for Statistical Computing. Vienna, Austria: R Foundation for Statistical Computing; 2013.

79. Royston JP. Algorithm AS 181: the W test for normality. J R Stat Soc C (Appl Stat). 1982;31:176-80.

80. Box JF. Guinness, Gosset, Fisher, and Small Samples. 1987. p. 45-52.

81. Bauer DF. Constructing confidence sets using rank statistics. J Am Stat Assoc. 1972:67:687-90.

82. Warnes GR, Bolker B, Bonebakker L, Gentleman R, Huber W, Liaw A et al. gplots: Various R Programming Tools for Plotting Data. R package version 2. 16.0. 2015. http://cran.r-project.org/package=gplots.

83. Neuwirth E. RColorBrewer: ColorBrewer Palettes. R package version 1.1-2. 2014. http://cran.r-project.org/package=RColorBrewer.

84. Chopra S, Hoshino A, Boddu J, lida S. Flavonoid Pigments as Tools in Molecular Genetics. In: Grotewold E, editor. The Science of Flavonoids. New York: Springer; 2006. p. 147-73.

85. Caspi $R$, Altman $T$, Dreher $K$, Fulcher CA, Subhraveti $P$, Keseler IM, et al. The MetaCyc database of metabolic pathways and enzymes and the BioCyc collection of pathway/genome databases. Nucleic Acids Res. 2012;40:D742-D53. 